\title{
Prevalência dos casos de Câncer de próstata e mama em Governador Valadares, Minas Gerais, Brasil (2009-2014)
}

\author{
Prevalence of prostate and breast cancer in Governador Valadares, Minas Gerais, Brazil \\ (2009-2014)
}

Prevalencia de los casos de Cáncer de próstata y mama en Gobernador Valadares, Minas Gerais, Brasil (2009-2014)

Lourimar Viana Nascimento Franco de Sousa ${ }^{1}$, Gisele Maria de Sousa ${ }^{2}$, Bernard Pereira de Almeida $^{3}$, José Maurício Diascânio ${ }^{4}$, Priscila Carla Souza da Mata ${ }^{1}$, Ronne Sangi da Silva ${ }^{1}$, Brenda Syan Mendes ${ }^{1}$, Eliana Martins ${ }^{1}$, Suelen dos Santos Ferreira ${ }^{5}$, Elder Oliveira da Silva ${ }^{6 *}$.

\section{RESUMO}

Objetivo: Descrever a prevalência dos cânceres de próstata e mama diagnosticados no Núcleo de Especialistas em Oncologia de Governador Valadares, Minas Gerais de setembro de 2009 a dezembro de 2014. Método: Estudo epidemiológico, retrospectivo, descritivo, quantitativo e transversal. Resultados: Dos 2.060 pacientes $51 \%$ foram diagnosticados câncer de próstata e $49 \%$ de mama. A maioria possui o ensino fundamental incompleto e são de municípios próximos. A faixa etária predominante para o câncer de mama foi de 50-59 anos e para o de próstata 70-79 anos. Em relação à predisposição familiar, ambos, não apresentaram antecedentes. Sobre os hábitos de vida, a maioria dos indivíduos nunca consumiram bebidas alcoólicas ou fumaram. Quanto ao estadiamento, conjuntamente as neoplasias encontram-se no Grau 2. Quanto à localização tumoral, no câncer de mama a maioria foi identificada no quadrante superior externo sendo a mama esquerda mais acometida. Em relação à letalidade, a frequência de óbitos para o câncer de mama foi de $16 \%$ e para o câncer de próstata foi de $11 \%$. Conclusão: A presente pesquisa reforça sobre a importância de mais investimentos e planejamento de ações estratégicas contra as neoplasias, possibilitando diagnóstico e tratamento precoce minimizando assim o número de mortes por essa enfermidade.

Palavras-Chave: Epidemiologia, Neoplasias, Câncer de Próstata, Câncer de mama.

\begin{abstract}
Objective: To describe the prevalence of prostate and breast cancers diagnosed at the Núcleo de Especialistas em Oncologia de Governador Valadares, Minas Gerais, from September 2009 to December 2014. Method: Epidemiological, retrospective, descriptive, quantitative and cross-sectional study. Results: Of the 2,060 patients, $51 \%$ were diagnosed with prostate cancer and $49 \%$ with breast cancer. Most have incomplete elementary education and are from nearby municipalities. The predominant age group for breast cancer was 50-59 years and for the prostate 70-79 years. Regarding the family predisposition, both had no antecedents. On lifestyle habits, most individuals never consumed alcohol or smoked. Regarding staging, the neoplasias are jointly found in Grade 2. Regarding the tumor location, in breast cancer the majority was

\footnotetext{
1 Universidade Vale do Rio Doce - UNIVALE. Governador Valadares-MG, Brasil.

2 Instituto Brasileiro de Educação Superior - IBES. Governador Valadares-MG, Brasil.

3 Serviço Nacional de Aprendizagem Comercial - SENAC. Vitória-ES, Brasil.

${ }^{4}$ Centro Federal de Educação Tecnológica do ES, CEFETES, Brasil.

${ }^{5}$ Universidad Columbia del Paraguay. Asuncion, Paraguay.

${ }^{6}$ Facultad de Ciencias Médicas. Universidad Privada del Guairá - UPG. Ciudad del Este, Paraguay. *E-mail: elder.enf@hotmail.com
} 
identified in the external superior quadrant, the left breast being more affected. Regarding the lethality, the frequency of deaths for breast cancer was $16 \%$ and for prostate cancer was $11 \%$. Conclusion: The present research reinforces the importance of more investments and strategic planning against neoplasias, allowing diagnosis and early treatment, thus minimizing the number of deaths due to this disease.

Keywords: Epidemiology, Neoplasms, Prostate cancer, Breast cancer.

\section{RESUMEN}

Objetivo: Describir la prevalencia de los cánceres de próstata y mama diagnosticados en el Núcleo de Especialistas en Oncología de Gobernador Valadares, Minas Gerais de septiembre de 2009 a diciembre de 2014. Método: Estudio epidemiológico, retrospectivo, descriptivo, cuantitativo y transversal. Resultados: De los 2.060 pacientes $51 \%$ fueron diagnosticados cáncer de próstata y $49 \%$ de mama. La mayoría posee la enseñanza fundamental incompleta y son de municipios cercanos. El grupo de edad predominante para el cáncer de mama fue de 50-59 años y el de próstata 70-79 años. En relación a la predisposición familiar, ambos, no presentaron antecedentes. Sobre los hábitos de vida, la mayoría de los individuos nunca consumieron bebidas alcohólicas o fumaron. En cuanto a la estadificación, conjuntamente las neoplasias se encuentran en el Grado 2. En cuanto a la localización tumoral, en el cáncer de mama la mayoría fue identificada en el cuadrante superior externo siendo la mama izquierda más acometida. En relación a la letalidad, la frecuencia de muertes por cáncer de mama fue del $16 \%$ y el cáncer de próstata fue del $11 \%$. Conclusión: La presente investigación refuerza sobre la importancia de más inversiones y planificación de acciones estratégicas contra las neoplasias, posibilitando diagnóstico y tratamiento precoz minimizando así el número de muertes por esa enfermedad.

Palabras clave: Epidemiología, Neoplasias, Cáncer de próstata, Cáncer de mama.

\section{INTRODUÇÃO}

De acordo com a Organização Mundial da Saúde (OMS), o câncer é um grave problema de saúde pública devido à sua ampla magnitude, sendo mais relevante entre os países em desenvolvimento, sendo estimado até 2025 mais de 20 milhões de casos novos (INCA, 2017; SARRI AJ, et al., 2017).

Na população brasileira o câncer é a segunda causa de morte, estando atrás somente das doenças cardiovasculares (INCA, 2017).

O câncer de mama é considerado o segundo tipo de câncer mais comum no mundo, sendo o mais frequente entre as mulheres (SCHILLING MPR, et al., 2017).

É um dos mais temidos, devido à sua alta frequência e seus efeitos. O câncer de mama pode afetar a vida da mulher nos aspectos psicológicos e sexuais, uma vez que os traumas do mesmo podem interferir em sua autoestima e provocar o medo de rejeição (SARRI AJ, et al., 2017)

Os sintomas mais relevantes inicialmente são dor mamária, alterações da pele, abaulamentos ou retrações com aspecto semelhante à "casca de laranja" e nódulo na mama e/ou axila. Os cânceres de mama estão localizados, principalmente, no quadrante superior externo, e em geral, as lesões são indolores, fixas e com bordas irregulares, acompanhadas de alterações da pele quando em estádio avançado (SILVA PA e RIUL SS, 2011).

No Brasil, o acometimento se faz em idade média de 52 anos (GONÇALVES LLC, et al., 2010). História familiar de câncer de mama, principalmente, em parentes de $1^{\circ} \mathrm{grau}$, tem sido associada a um elevado risco de desenvolver essa doença.

Observa-se um risco aumentado nas mulheres com menos de 40 anos de idade, naquelas com histórico familiar de câncer de mama na pré-menopausa, quando o câncer for bilateral ou a recorrência familiar atingir dois ou mais parentes de primeiro grau (PIVETTA HMF et al. 2015). 
Outro fator relacionado na literatura é a obesidade. O excesso de peso corporal parece influenciar no desenvolvimento e na progressão do câncer de mama devido ao aumento da síntese do estrógeno, resistência à insulina e ativação de vias inflamatórias (SILVA PA e RIUL SS, 2011).

No Brasil, o câncer de próstata é o segundo mais comum entre os homens, atrás apenas do câncer de pulmão (INCA, 2017). Com o envelhecimento, a tendência é que a próstata aumente de tamanho, dessa forma tornando o fluxo urinário lento e mais difícil para indivíduos a partir dos 50 anos, isso ocorre devido à compressão da uretra dificultando assim a passagem da urina, o que torna o jato urinário gradativamente fino e fraco (INCA, 2017). A próstata é importante na prática urológica, pois apresenta ligação a vários processos que causam transtornos a pacientes de diferentes idades (LUU HN, et al., 2017).

Estudos apontam que a próstata tem atividade de produzir $50 \%$ dos fluidos que compõem o sêmen, tendo uma função biológica importante na fase reprodutora masculina (OBATA A, et al., 2017).

O câncer da próstata pode apresentar evolução silenciosa, inicialmente, os pacientes podem estar assintomáticos ou, apresentarem sintomas parecidos aos do tumor benigno da próstata, como por exemplo, dificuldade para urinar. Com o avanço da doença, podem apresentar dor óssea, problemas urinários, infecção generalizada ou insuficiência renal (BACELAR JA, et al., 2015).

No câncer de próstata a idade constitui um fator de risco considerável assim como nos outros tipos de câncer, visto que, tanto a incidência quanto a mortalidade tem um aumento significativo após os 50 anos. Indivíduos com histórico familiar de câncer da próstata antes dos 60 anos, é um marcador importante que pode aumentar de 3 a 10 vezes o risco de desenvolver essa enfermidade (SCHILLING MPR, et al., 2017).

Outros fatores cujas associações com câncer da próstata foram detectadas são o consumo excessivo de álcool, tabagismo e a vasectomia (OBATA A, et al., 2017).

Considerando que há um elevado índice de câncer de próstata e mama na população brasileira, tem-se a necessidade de conhecer mais sobre o acometimento desses cânceres. O objetivo da presente pesquisa foi descrever a prevalência dos cânceres de próstata e mama diagnosticados por biópsia no Núcleo de Especialistas em Oncologia de Governador Valadares, estado de Minas Gerais, no período compreendido entre setembro de 2009 a dezembro de 2014.

\section{METODOLOGIA}

Estudo epidemiológico, retrospectivo, descritivo, quantitativo e transversal, com amostragem nãoprobabilística por conveniência, realizado em 2017 no Núcleo de Especialistas em Oncologia de Governador Valadares estado de Minas Gerais.

Como critério de inclusão, foi considerado todo paciente que realizou consulta neste Núcleo e apresentou diagnóstico confirmado por biópsia para o câncer de próstata e de mama no período de setembro de 2009 a dezembro de 2014. Excluíram-se desta pesquisa, pacientes que realizam atendimento médico nesse período, porém, com resultado de biópsia negativo.

Os dados foram coletados no período de 9 de janeiro a 3 de julho de 2017, onde foram analisados 2.060 prontuários, sendo, 1.014 para câncer de mama e 1.046 para o câncer de próstata. Este estudo foi aprovado pelo Comitê de Ética em Pesquisa da Universidade Vale do Rio Doce (UNIVALE) / FUNDAÇÃO PERCIVAL FARQUHAR (FPF) sob o parecer de oㅜ 1.800.850 e CAEE 60723816.3.0000.5157. Para tabulação dos dados, foi utilizado o programa da Microsoft $\Theta$ Excel versão 2013 e, posteriormente, calculadas as frequências absolutas e relativas.

\section{RESULTADOS E DISCUSSÃO}

Foram analisados 2.060 prontuários de pacientes com diagnóstico de câncer confirmado por biopsia (Figura 1). No período compreendido entre setembro de 2009 a dezembro de 2014 foram diagnosticados 51\% (1.046) indivíduos com câncer de próstata e 49\% (1.014) com câncer de mama. 
Figura 1 - Prevalência dos pacientes com câncer de próstata e mama diagnosticados por biópsia no Núcleo de Especialistas em Oncologia de Governador Valadares em Governador Valadares, Minas Gerais, Brasil (2009-2014).

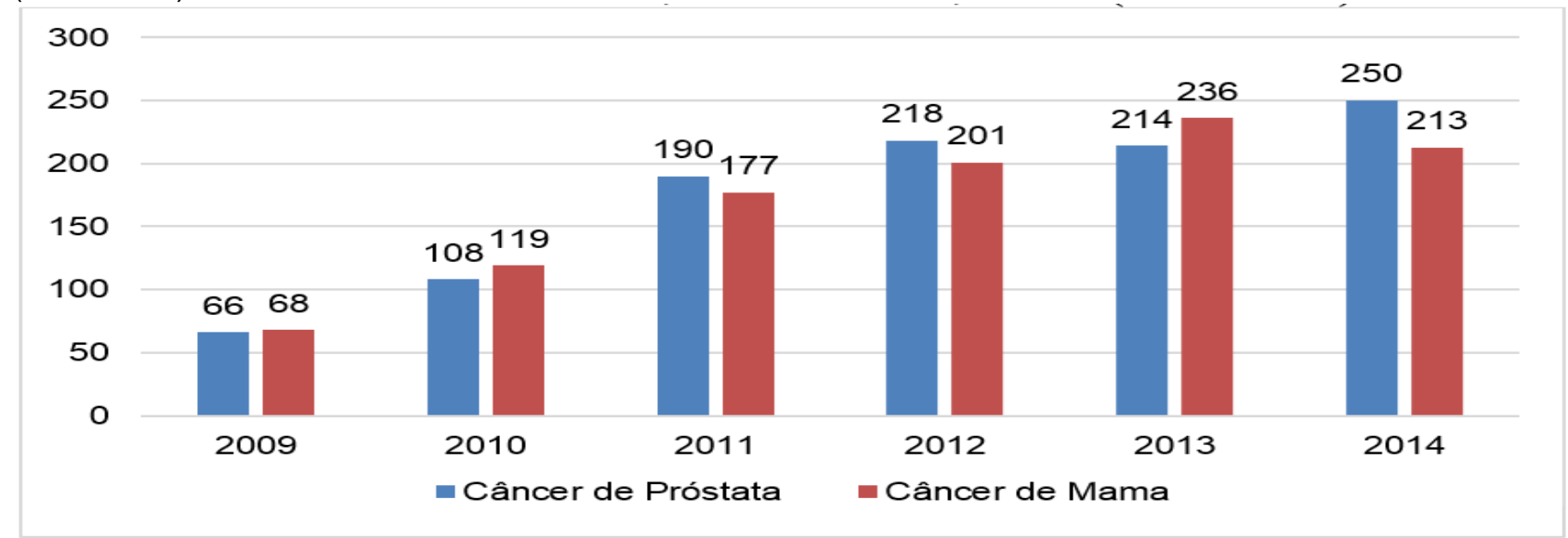

Fonte: Dados da pesquisa (2017).

Percebeu-se o aumento considerável de pessoas diagnosticadas com câncer de próstata e mama nos últimos tempos entre os anos de 2009 a 2014. Em 2009, foi observado que o número de casos diagnosticados foi menor, isso se justifica pelo fato desta instituição ter sido inaugurada em setembro deste mesmo ano. $O$ aumento no número de casos para os anos seguintes possivelmente pode ser justificado pelo fato das pessoas terem mais acesso aos meios de comunicação. Isso tem facilitado a conscientização das pessoas sobre a importância da prevenção contra o câncer. Diante disso, o número de casos vem aumentando porem, sendo descobertos em estágios mais precoces.

Tabela 1 - Variáveis sociodemográficas e caracterização dos pacientes com câncer de próstata e mama diagnosticados por biópsia no Núcleo de Especialistas em Oncologia de Governador Valadares em Governador Valadares, Minas Gerais, Brasil (2009-2014).

\begin{tabular}{lcccc} 
& Variáveis & \multicolumn{2}{c}{ Câncer de Próstata } & \multicolumn{2}{c}{ Câncer de Mama } \\
\cline { 2 - 5 } & $\mathbf{n}$ & $\%$ & $\mathbf{n}$ & $\%$ \\
\hline Escolaridade & & & & \\
Nenhuma & 199 & 19 & 128 & 13 \\
Fundamental Incompleto & 262 & 25 & 277 & 27 \\
Fundamental Completo & 82 & 8 & 105 & 10 \\
Nível Médio & 79 & 7 & 118 & 11 \\
Superior Incompleto & 4 & 1 & 13 & 1 \\
Superior Completo & 16 & 2 & 122 & 13 \\
Sem Informação & 404 & 38 & 251 & 25 \\
Faixa etária & & & & \\
De 10 a 19 anos & 0 & 0 & 1 & 1 \\
De 20 a 29 anos & 0 & 0 & 12 & 3 \\
De 30 a 39 anos & 2 & 1 & 79 & 8 \\
De 40 a 49 anos & 12 & 3 & 216 & 20 \\
De 50 a 59 anos & 111 & 11 & 267 & 25 \\
De 60 a 69 anos & 344 & 31 & 241 & 23 \\
De 70 a 79 anos & 444 & 40 & 139 & 13 \\
De 80 a 89 anos & 126 & 12 & 54 & 5 \\
De 90 a 99 anos & 7 & 2 & 5 & 2 \\
Histórico Familiar & & & & \\
Com histórico & 278 & 26 & 440 & 43 \\
Sem histórico & 730 & 70 & 564 & 56 \\
Sem informação & 38 & 4 & 10 & 1 \\
\hline Fon Dados dapesquisa
\end{tabular}

Fonte: Dados da pesquisa (2017). 
A importância do diagnóstico precoce ser divulgado de forma tão intensa, acabou com o pensamento de que o grande número de mulheres com tumores avançados no Brasil era por causa da falta de conscientização e por receio de receberem o diagnóstico da doença (ANTON E, et al., 2015). A alta divulgação nas mídias vem provocando a cancerofobia em jovens pois, mesmo sabendo que a neoplasia é rara antes dos 35 anos (SCHILLING MPR, et al., 2017).

Segundo grau de instrução, predominou o ensino fundamental incompleto tanto para o câncer de mama como para o câncer de próstata 25\% (262) e 27\% (277) respectivamente (Tabela 1).

A baixa escolaridade tem forte influência na prevenção do câncer e consequentemente na detecção precoce do tumor (SCHILLING MPR, et al., 2017). Em ambos os sexos, a maioria não possui o ensino fundamental. Pacientes menos esclarecidos procuram com menor frequência às consultas. Portanto, não conhecem a importância da prevenção, dos exames, e assim não adquirem as informações necessárias sobre a doença, o que leva a um diagnóstico tardio (KOOTSTRA JJ, et al., 2013).

Há relatos que a baixa escolaridade interfere de forma secundária na prevenção do câncer de mama, pois, mulheres com esse perfil, muitas vezes não realizam o auto exame e procuram menos o serviço de saúde para realizarem o exame clínico e a mamografia (AVELAR E, et al 2017; ALBRECHT CAM, et al., 2013).

A faixa etária com maior acometimento para o câncer de mama foi de 50 a 59 anos com 25\% (267) casos, porém, o número de mulheres com diagnóstico positivo para este tumor começa a crescer a partir dos 30 anos. Para o câncer de próstata, a faixa etária predominante foi a de 70 a 79 anos com $40 \%$ (444) casos, tendo um aumento significativo a partir dos 50 anos.

No presente estudo, o diagnóstico de câncer de mama foi maior entre mulheres com idade entre 50 e 59 anos e em homens, o câncer de próstata foi maior entre 70 e 79 anos. Em outro estudo desenvolvido em mulheres na Amazônia, a faixa etária mais comum foi entre 40 a 49 anos (SCHILLING MPR, 2017). Estudos sustentam a hipótese de que o organismo a partir das faixas etárias citadas é mais suscetível a tumores devido ao envelhecimento celular e a diminuição da capacidade das células de se recuperar (ANTON E, et al., 2015).

Em relação à predisposição, para o câncer de próstata 26\% (278) apresentaram histórico familiar, 70\% (730) não possuíam relatos na família e 4\% (38) não consta a informação no prontuário. Para o câncer de mama 43\% (440) possuem histórico familiar, 56\%(564) não apresentam casos na família e 1\% (10) não consta a informação no prontuário.

As neoplasias não possuem uma única causa, mas sim, diversos fatores que estão interligados com a probabilidade de desenvolver a doença, tais como: idade, histórico familiar, fatores comportamentais / ambientais como uso de bebidas alcoólicas e tabaco, dentre outros (OBATA A, 2017).

Na presente pesquisa, tanto para os pacientes com câncer de mama quanto de próstata, a maioria não possui histórico familiar de neoplasias. Schilling MPR, et al. (2017) destaca que a predisposição familiar para a neoplasia da mama em parentes de primeiro grau está associado a um risco elevado.

A probabilidade de se ter um câncer aumenta nas mulheres com menos de 40 anos, quando existem familiares próximos com ocorrência de câncer de mama na pré-menopausa, quando este for bilateral ou quando a recorrência atingir dois ou mais parentes $(\mathrm{OH} \mathrm{H}$, et al., 2015).

O histórico familiar de câncer de próstata é um fator de risco reconhecido para a doença, por isso, é recomendável um rastreamento de histórico em torno dos 45 anos (McTIERNAN A, 2003).

Para Anjos et al. (2016) os riscos em relação ao histórico familiar e a incidência do câncer de próstata aumentam 2,2 vezes quando é um parente de primeiro grau, 4,9 vezes quando dois parentes de primeiro grau desenvolvem a doença e 10,9 vezes quando três parentes de primeiro grau apresentam o tumor. Portanto, quando uma pessoa possui um histórico familiar de câncer, independente se é de mama ou próstata, por questões de probabilidade, é recomendável que esta pessoa busque informações sobre a doença e faça consultas regularmente. 
Ao verificar a procedência, constatou-se que a maioria é proveniente de outras cidades, sendo que para ambas as neoplasias, 48\% (985) são pertencentes ao município de Governador Valadares e 52\% (1.075) são de outros municípios próximos.

Dos 1.014 casos de câncer de mama 49\% (504) dos pacientes residiam em Governador Valadares e os outros $51 \%$ (510) pertenciam às outras cidades. Para o câncer de próstata dos 1.046 casos $46 \%$ (481) eram de Governador Valadares e 54\% (565) eram moradores das cidades próximas.

Esse evento ocorre pelo fato de Governador Valadares ser a maior cidade da macrorregião do leste de Minas Gerais, possuindo assim, o maior número de centros especializados para o tratamento oncológico, o que atrai pacientes de outras localidades.

Tabela 2 - Hábitos de vida dos pacientes com câncer de próstata e mama diagnosticados por biópsia no Núcleo de Especialistas em Oncologia de Governador Valadares em Governador Valadares, Minas Gerais, Brasil (2009-2014).

\begin{tabular}{|c|c|c|c|c|c|c|c|c|}
\hline \multirow{3}{*}{ Variáveis } & \multicolumn{4}{|c|}{ Portadores câncer de próstata } & \multicolumn{4}{|c|}{ Portadores câncer de mama } \\
\hline & \multicolumn{2}{|c|}{ Etilismo } & \multicolumn{2}{|c|}{ Tabagismo } & \multicolumn{2}{|c|}{ Etilismo } & \multicolumn{2}{|c|}{ Tabagismo } \\
\hline & $\mathbf{n}$ & $\%$ & $\mathbf{n}$ & $\%$ & $\mathbf{n}$ & $\%$ & $\mathbf{n}$ & $\%$ \\
\hline Nunca consumiu & 692 & 66 & 637 & 61 & 938 & 93 & 825 & 81 \\
\hline Ex consumidor & 109 & 10 & 187 & 18 & 12 & 1 & 65 & 7 \\
\hline Consumidor & 229 & 22 & 207 & 20 & 55 & 5 & 115 & 11 \\
\hline Sem informação & 16 & 2 & 15 & 1 & 9 & 1 & 9 & 1 \\
\hline
\end{tabular}

Fonte: Dados da pesquisa (2017).

No quesito hábitos de vida, a maioria dos indivíduos com câncer de próstata 66\% (692) e de mama 93\% (938) nunca consumiram bebidas alcoólicas, 10\%(109) homens e 1\%(12) mulheres se declararam exconsumidores e $22 \%$ (229) dos que possuem o câncer de próstata e 5\%(55) câncer de mama declararam etilistas.

Resultado aproximado foi encontrado para o consumo de tabaco, onde nos casos de câncer de próstata, $61 \%$ (637) dos pacientes nunca fumaram, 18\% (187) eram ex consumidores e 22\% (229) ainda eram usuários. Com relação aos portadores de câncer de mama $81 \%$ (825) nunca consumiram tabaco, 7\% (65) são exfumantes, $11 \%$ (115) ainda continuam fumando.

O uso de bebidas alcoólicas e tabaco são considerados fatores predisponentes ao câncer (SCHILLING MPR, et al., 2017; SAFARI A, 2016). Dos 1.046 homens e das 1.014 mulheres diagnosticados com câncer, $60 \%$ e $81 \%$ respectivamente, nunca fizeram uso de bebidas alcoólicas.

Mesmo com este elevado número de pessoas que nunca ingeriram bebidas alcoólicas é recomendado a não utilização dos mesmos, pois para aqueles com pré-disposição a sua utilização pode desencadear a doença (SAFARI A, 2016).

O uso do tabaco pode desencadear o câncer principalmente no pulmão, na cavidade oral, laringe, faringe e esôfago (SANTIS CE, et al., 2015).

Ao avaliar os prontuários dos pacientes, foi observado que um elevado número de pessoas nunca havia feito uso do tabaco. De 1.046 homens e 1.014 mulheres a maioria declarou nunca ter consumido tabaco. Os dados analisados no presente estudo mostraram que o tabaco não teve influência no surgimento do câncer de próstata e mama.

Considerando o grau de estadiamento, constatou-se tanto no câncer de próstata quanto para o de mama que a maioria dos casos representado por 51\% (1.057) encontrava-se no Grau 2 (Figura 2). 
Figura 2 - Grau de estadiamento do tumor nos pacientes com câncer de próstata e mama diagnosticados por biópsia no Núcleo de Especialistas em Oncologia de Governador Valadares em Governador Valadares, Minas Gerais, Brasil (2009-2014).

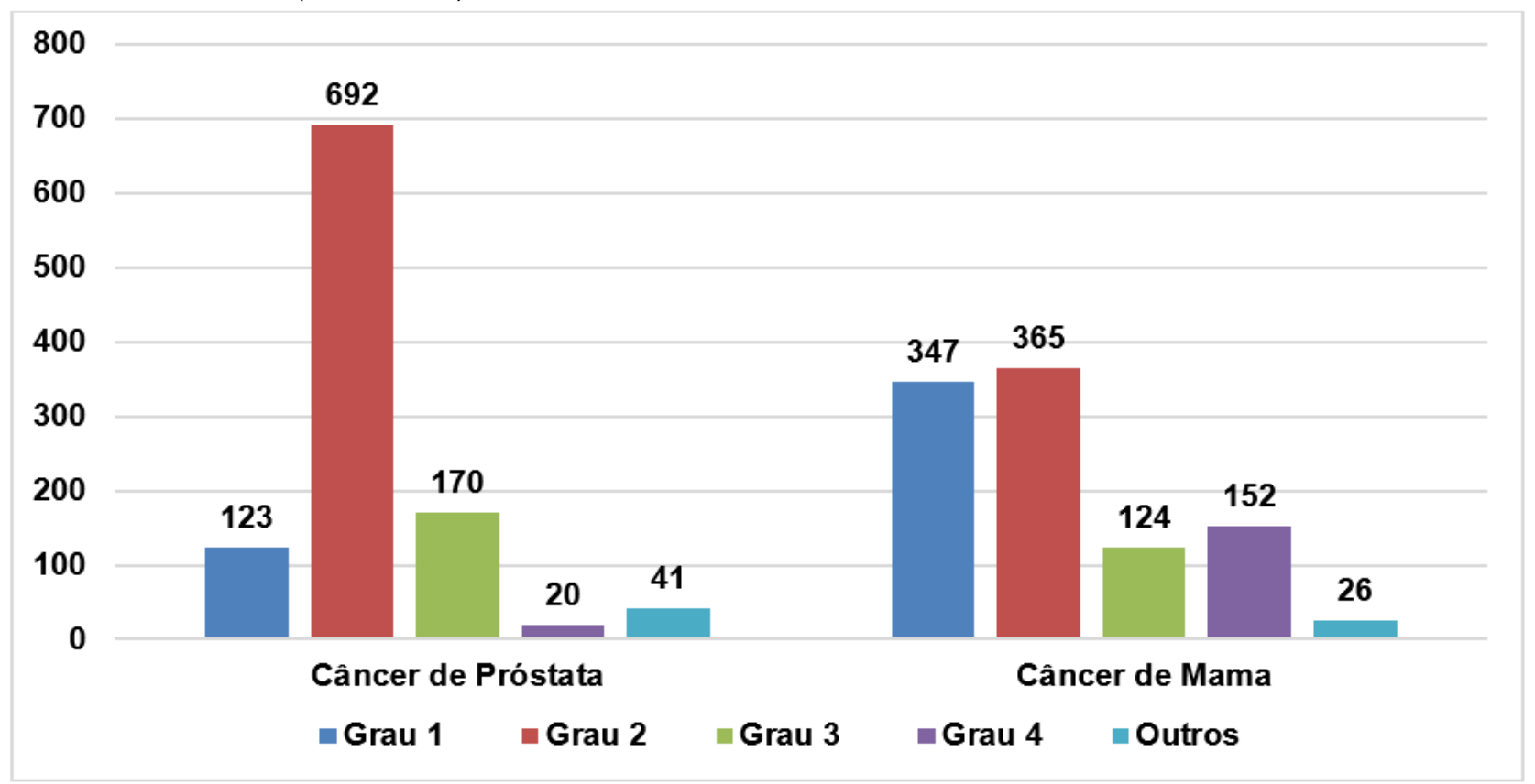

Fonte: Dados da pesquisa (2017).

O estadiamento é definido como um sistema TNM (tumor/nódulo/metástases) usado para descrever e registrar a extensão anatômica da doença que consiste na classificação dos casos de neoplasia maligna, de forma a avaliar seu grau de disseminação (HABRAKEN V, et al., 2017)

O procedimento para verificação do estadiamento é o recomendado pela União Internacional Contra o Câncer (UICC), intitulado Sistema TNM de Classificação dos Tumores Malignos. O "T" observa o aspecto do tumor primário, "N" o comprometimento dos linfonodos e o "M" é o aparecimento ou não de metástases.

Este modelo possui ordenações graduais, geralmente de T0 a T4, de N0 a N3 e de M0 a M1, nessa ordem (HABRAKEN V, et al., 2017; INCA, 2017). Após a avaliação destes fatores, os casos são classificados em grau 1, 2, 3, 4 .

As categorias T e N, além das classificações numéricas, podem ser subclassificadas em letras (a,b e c), de forma a expressar a evolução do tumor e dos linfonodos comprometidos. Quando na classificação surge o símbolo "X" quer dizer que a categoria não pode ser devidamente avaliada (INCA, 2017).

No presente estudo, predominou o estadiamento Grau 2, sendo 66\% (692) para câncer de próstata e 36\% (365) para o câncer de mama. Neste estágio da doença, a mesma é avaliada como tendo um bom prognostico, pois nesse estágio, ainda são considerados como pertencendo à fase inicial, o que aumenta a chance de cura.

Segundo o Instituto Nacional do Câncer (2017), os tumores cancerosos podem surgir em qualquer parte da mama, porém, em quase $50 \%$ dos casos o quadrante superior externo, é a região frequentemente mais acometida. Os dados encontrados na pesquisa corroboram com a literatura e outros estudos (HABRAKEN V, et al., 2017; INCA, 2017; FERLAY J, 2015; NAKASHIMA J, et al., 2012).

Ao analisar a lateralidade do câncer de mama, 52\% (525) apresentaram o tumor na mama esquerda, 45\% (458) na mama direita, 2\% (23) nas duas mamas e 1\% (8) não consta a informação no prontuário.

Quanto à localização do tumor primário em 35\% (354) casos, foram localizados no quadrante superior externo independente da mama (Figura 3). 
Figura 3 - Localização do tumor primário pacientes com câncer de próstata e mama diagnosticados por biópsia no Núcleo de Especialistas em Oncologia de Governador Valadares em Governador Valadares, Minas Gerais, Brasil (2009-2014).

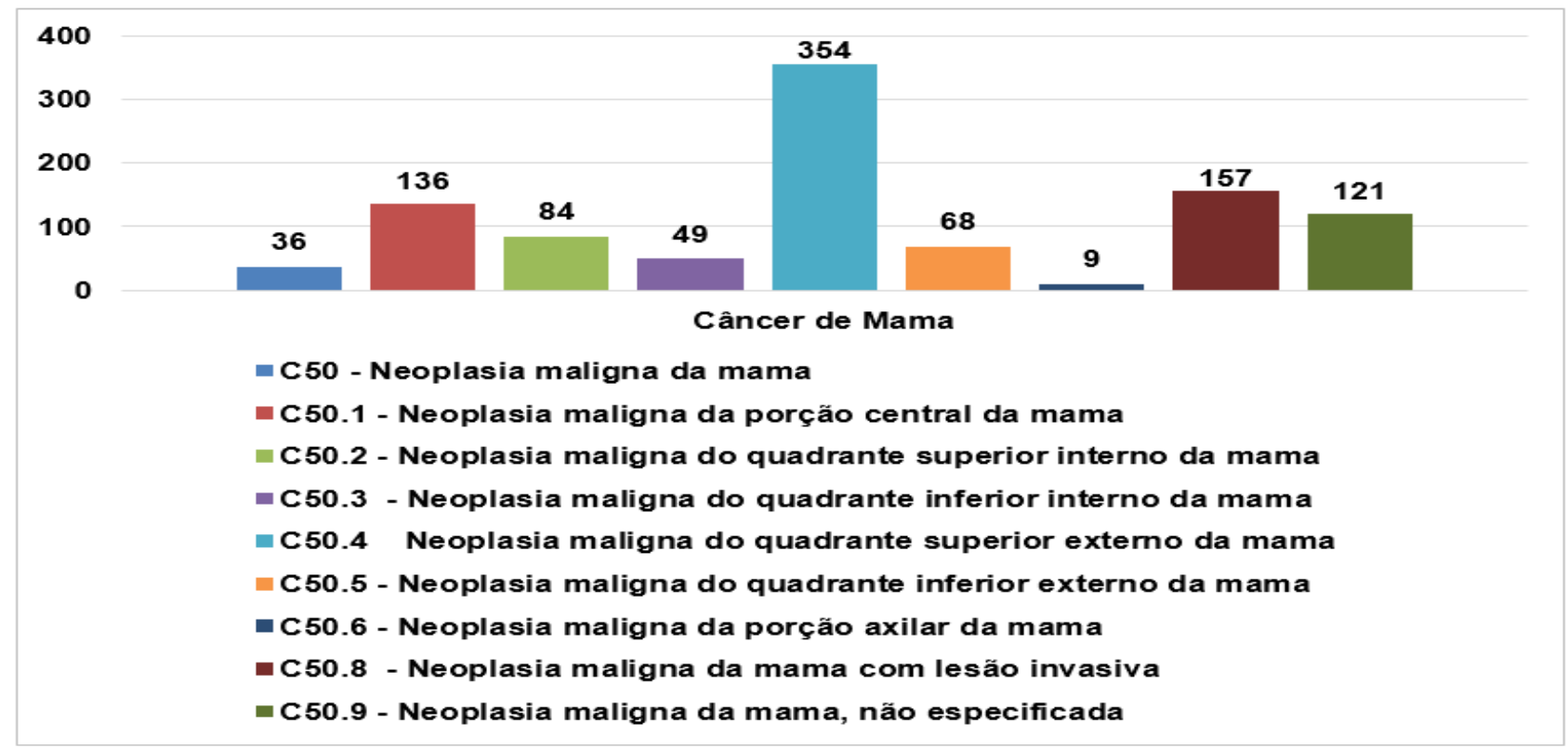

Fonte: Dados da pesquisa (2017).

Figura 4 - Lateralidade do tumor primário nos pacientes com câncer de próstata e mama diagnosticados por biópsia no Núcleo de Especialistas em Oncologia de Governador Valadares em Governador Valadares, Minas Gerais, Brasil (2009-2014).

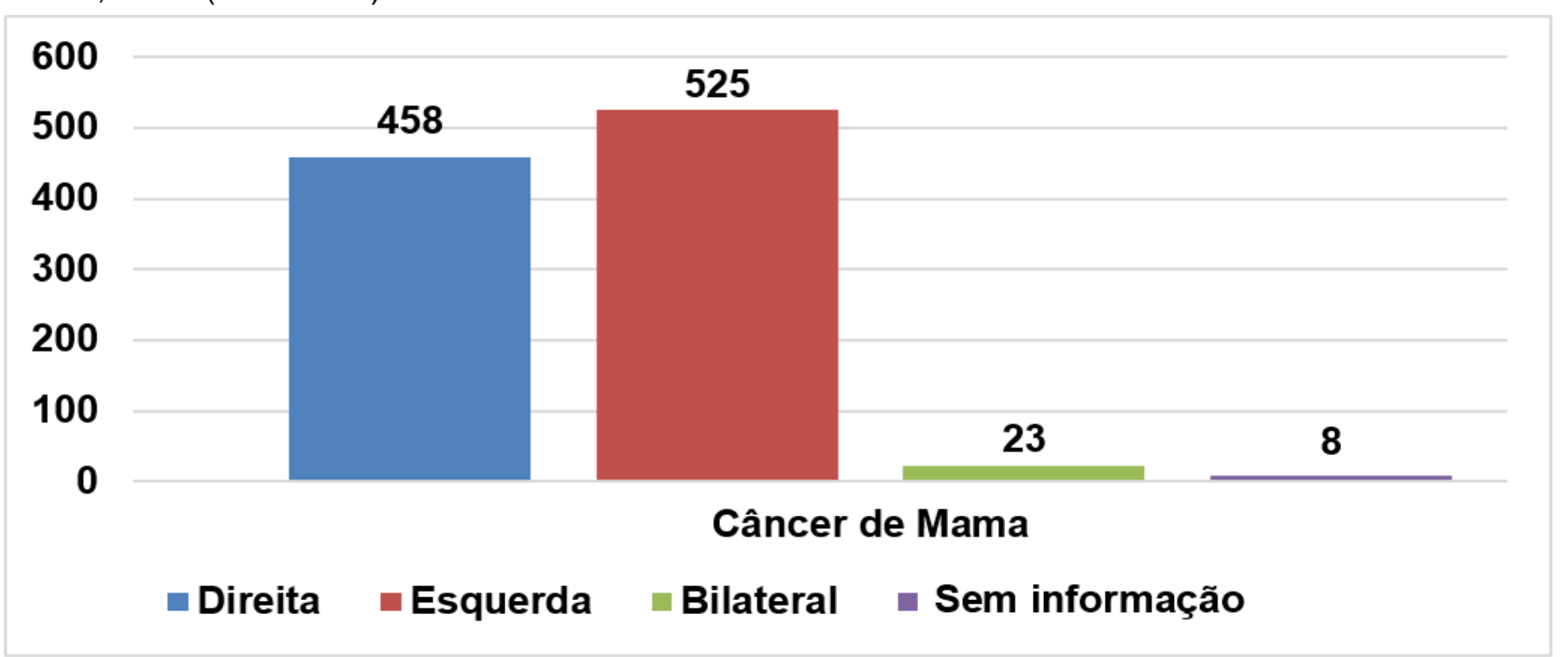

Fonte: Dados da pesquisa (2017).

A lateralidade da neoplasia na mama é um fator relevante a ser considerado no cálculo de risco, pois existe a importância de se conhecer o local do tumor e o tipo, porque podem variar em seu grau de gravidade (FERLAY J, 2015; NAKASHIMA J, et al., 2012).

Para Pinheiro et al. (2013), a lateralidade constitui um dos fatores determinantes na melhor forma de abordagem para a realização da incisão. Estudos apontam que pacientes que apresentam tumoração na mama direita, apresentam maior frequência de metástase para os linfonodos axilares, sendo necessário a realização de mastectomia total com esvaziamento axilar ganglionar (BARROS, et al.,2013; PINHEIRO, et at., 2013; NAKASHIMA J, et al., 2012) 
A frequência de óbitos para o câncer de mama foi de 16\% (162) mortes, e para o câncer de próstata foi de $11 \%(115)$ (Tabela 2).

Tabela 2 - Frequência de óbitos nos pacientes diagnosticados com câncer de câncer de próstata e mama em Governador Valadares, Minas Gerais, Brasil (2009-2014).

\begin{tabular}{lcccccc} 
& \multicolumn{2}{c}{ Câncer de Mama } & \multicolumn{2}{c}{ Câncer de Próstata } & \multicolumn{2}{c}{ Total } \\
\cline { 2 - 7 } & $\mathbf{n}$ & $\%$ & $\mathbf{n}$ & $\%$ & $\mathbf{n}$ & $\%$ \\
\hline Óbitos & 162 & 16 & 115 & 11 & 277 & 13 \\
Sobreviventes & 852 & 84 & 931 & 89 & 1783 & 87 \\
\hline
\end{tabular}

Fonte: Dados da pesquisa (2017).

O câncer de mama é uma das doenças que provocam mais mortes entre as mulheres brasileiras (SCHILLING MPR, et al., 2017; AVELAR E, 2017; NAKASHIMA J, et al., 2012). O câncer de próstata é a segunda maior causa de morte no país entre homens, ficando atrás apenas do câncer de pulmão (SCHILLING MPR, et al., 2017)

No presente estudo, do total de 1.014 mulheres com câncer de mama 16\% (162) foram a óbito, e dos 1.046 com câncer de próstata 11\% (115) faleceram. O baixo índice de óbitos pode estar relacionado ao diagnóstico realizado no estágio inicial do câncer com isso, proporcionando o início rápido do tratamento.

\section{CONCLUSÃO}

Os resultados aqui apresentados revelam um importante aumento no número de casos de câncer de próstata e mama em indivíduos de Governador Valadares e municípios próximos. Sugerimos maior investimento na realização de ações preventivas dentro da atenção primaria, principalmente na população menos instruída, buscando assim uma melhor qualidade de vida para a população.

\section{REFERÊNCIAS}

1. ALBRECHT CAM, et al. Breast cancer mortality among patients attending a cancer hospital, Vitoria, ES. Rev Bras Epidemiol 2013; 16(3): 582-591.

2. ANJOS QDS, et al. Práticas de Prevenção do Câncer de Próstata em Uma Unidade Básica de Saúde no Município de Rolim de Moura-RO. Revista Enfermagem e Saúde Coletiva 2016; 1(2):2-18.

3. ANTON E, et al. The importance of clinical and instrumental diagnostic in the mammary gland cancer. Rev Med Chir Soc Med Nat lasi 2015; 119(2):410-418.

4. AVELAR E, STRICKLAND CR, ROSITO G. Role of Imaging in Cardio-Oncology. Curr Treat Options CardiovasC Med. 2017; 19(6):46.

5. BACELAR JA, et al. Câncer De Próstata: Métodos De Diagnóstico, Prevenção E Tratamento. Braz J Surg Clin Res 2015; 10(3):40-46.

6. BARROS, et al. Tempo para acesso ao tratamento do câncer de mama no Distrito Federal, Brasil Central. Rev Bras Ginecol Obstet. 2013; 35(10):458-63.

7. De SANTIS CE, et al. International variation in female breast cancer incidence and mortality rates. Cancer Epidemiol Biomarkers Prev2015; 24(10):1495-1506.

8. FERLAY J, et al. Cancer incidence and mortality worldwide: Sources, methods and major patterns in GLOBOCAN 2012: Globocan 2012. Int J Cancer 2015; 136(5): 359-86.

9. GONÇALVES LLC, et al. Breast cancer risk factors in women receiving care at a cancer out patient department. Rev Enferm UERJ 2010; 18(3):468-72.

10. HABRAKEN V, et al. Does the TNM classification of solitary internal mammary lymph node metastases in breast cancer still apply?. Breast Cancer Res Treat 2017; 161:483-489.

11. INSTITUTO NACIONAL DO CÂNCER (Brasil). Estimativas da incidência e mortalidade por câncer 2016. Rio de Janeiro: INCA; 2017.

12. KOOTSTRA JJ, et al. A longitudinal study of shoulder and arm morbidity in breast cancer survivors 7 years after sentinel lymph node biopsy or axillary lymph node dissection. Breast Cancer Res Treat2013; 139(1):125-134. 
13. Luu HN, et al. Mirnas associated with prostate cancer risk and progression. BMC Urology 2017; 17(1):1-18.

14. MCTIERNAN A. Behavioral risk factors in breast cancer: can risk be modified?. Oncologist 2003; 8, 326-34.

15. NAKASHIMA J, et al. Cancer incidence in the Western Amazon: population-based estimates in Rio Branco, Acre State, Brazil, 2007-2009. Cad Saude Publica 2012; 28: 2125-32.

16. OBATA A, et al. Advanced breast cancer in a relatively young man with severe obesity and type 2 diabetes mellitus. J Diabetes Investig. 2017; 8(3):395-396.

17. $\mathrm{OH} \mathrm{H}$, et al. The interaction between early-life body size and physical activity on risk of breast cancer.Int J Cancer 2015; 137(3):571-81.

18. PINHEIRO AB, et al. Câncer de Mama em Mulheres Jovens: Análise de 12.689 Casos. Revista Brasileira de Cancerologia 2013; 59(3): 351-359.

19. Pivetta HMF, et al. Perfil clínico patológico comparativo do câncer de mama em nulíparas e não nulíparas. Cadernos de educação, saúde e fisioterapia 2015; 2(3).

20. SARRI AJ, et al. Lymphoscintigraphy detecting alterations of upper limb lymphatic flow following early sentinel lymph node biopsy in breast cancer. Breast Cancer 2017; 19(9):279-285.

21. SCHILLING MPR, et al. Breast Cancer Awareness among Women in Western Amazon: a Population Based CrossSectional Study. Asian Pac J Cancer Prev 2017; 18(3):847-856.

22. SILVA PA, RIUL SS. Breast cancer: risk factors and early detection. Rev Bras Enferm 2011; 64(6):1016-102. 